\title{
Intelligent Artifact Classification for Ambulatory Physiological Signals
}

\author{
Kevin T Sweeney, Darren J Leamy, Tomás E Ward and Seán McLoone
}

\begin{abstract}
Connected health represents an increasingly important model for health-care delivery. The concept is heavily reliant on technology and in particular remote physiological monitoring. One of the principal challenges is the maintenance of high quality data streams which must be collected with minimally intrusive, inexpensive sensor systems operating in difficult conditions. Ambulatory monitoring represents one of the most challenging signal acquisition challenges of all in that data is collected as the patient engages in normal activities of everyday living. Data thus collected suffers from considerable corruption as a result of artifact, much of it induced by motion and this has a bearing on its utility for diagnostic purposes. We propose a model for ambulatory signal recording in which the data collected is accompanied by labeling indicating the quality of the collected signal. As motion is such an important source of artifact we demonstrate the concept in this case with a quality of signal measure derived from motion sensing technology viz. accelerometers. We further demonstrate how different types of artifact might be tagged to inform artifact reduction signal processing elements during subsequent signal analysis. This is demonstrated through the use of multiple accelerometers which allow the algorithm to distinguish between disturbance of the sensor relative to the underlying tissue and movement of this tissue. A brain monitoring experiment utilizing EEG and fNIRS is used to illustrate the concept.
\end{abstract}

\section{INTRODUCTION}

As life expectancy in the modern world continues to increase, the need for out-patient and in-home monitoring systems in the coming decades will rise considerably. The ability to monitor the patient in the comfort of their own home will greatly improve quality of life, while increasing the efficiency of health-care systems. These out-patient recordings can cost as little as 50\% of their inpatient counterparts [14], thus making them a more feasible option in many instances. In an increasing number of situations, the use of ambulatory recording techniques is the only approach available to detect the desired signals. Tests such as detecting epileptic seizures [11], cardiac arrhythmias [12] and sleepiness or drowsiness [8] often require the recording to occur in real world situations, and thus cannot be measured in a controlled environment. With this increased need for out-patient care, there is a corresponding increase in the

This work was supported in part by Irish Research Council for Engineering, Science and Technology (IRCSET) and Science Foundation Ireland: Research Frontiers Program 2009, Grant No. 09/RFP/ECE2376

$\mathrm{K}$. Sweeney is with the Department of Electronic Engineering, National University of Ireland Maynooth, Co. Kildare, Ireland email: ksweeneydeeng. nuim. ie

Darren Leamy, Tomás Ward and Seán McLoone are with the Department of Electronic Engineering, National University of Ireland Maynooth, Co. Kildare, Ireland email: tomas.warddeeng.nuim.ie sean.mcloonedeeng.nuim.ie

978-1-4244-4124-2/10/\$25.00 @2010 IEEE need for more accurate ambulatory recordings of the required physiological signals.

To date, there are a large number of available recording techniques including functional magnetic resonance imaging (fMRI), positron emission tomography (PET), singlepositron emission computed tomography (SPECT), magnetoencephalography (MEG), electroencephalography (EEG) and functional near-infrared spectroscopy (fNIR). Each technique bears its own individual advantages and disadvantages. These include spatial or temporal resolution, cost and ease of setup and use. However, only a few of these techniques are currently viable for use in a long term ambulatory setting.

This shift towards ambulatory recordings results in an increase in the interference embedded in the desired recorded physiological signal. Thus, as ambulatory physiological signal monitoring systems become more widespread, the need for techniques and algorithms capable of detecting these artifacts becomes fundamentally important. Post processing methods may allow amelioration of the effects of artifact but such methods must be properly informed of the nature of the artifact itself. Currently most ambulatory signal monitoring systems do not explicitly facilitate the collection of this additional contextualizing signal information.

The aim of this paper is to accurately and intelligently classify the physiological signals into separate epochs of data, determined by the nature of the artifact embedded on the signal. A short case study is then performed to demonstrate how the classifying algorithm operates, by use of a Quality of Signal (QOS) metric, and how using this classification can aid in the removal of the artifacts.

Section II of this paper describes the different forms of artifact enclosed in the physiological signals, section III explains how the embedded artifact is to be detected, section IV details the classification algorithm used, section V shows the findings of the classification algorithm by implementing some tests and section VI gives a brief overview of the findings of the paper.

\section{BIO-SIGNAL ARTIFACTS}

Artifacts are defined as being any unwanted variations in a signal caused by an external source. These artifacts can range from small insignificant changes in a signal to causing the desired signal to be indistinguishable from the noise. These artifacts can come in two distinct forms; biological noise and environmental noise [3]. Environmental noise originates from instruments and circuit components and can usually be removed by simple filtering techniques. 
Biological noise is artefact of a physiological origin. To date, there are a number of different techniques being used to remove the separate embedded artifacts. A comprehensive overview of some of the available methods can be found in [1]. Artifact due to the movement of the subject has a detrimental effect on the recorded signal as its spectrum often overlaps that of the desired signal and is therefore often difficult to remove. Consequently it is important to be able to accurately tag the clean recorded data as to retain as much good signal as possible and to employ the optimal artifact reduction technique where required.

\section{A. Motion Artifact}

Although environmental and biological noise are consistently a problem when recording physiological signals, the issue of subject motion only becomes significant in ambulatory recordings. In a clinical setting, motion can be kept to a minimum and any movement undertaken can be easily documented by the on-site recording technician.

As expected, when in motion, the body of the subject alters position which can interfere with measurement of the signal of interest being recorded. This can be observed for example, in photoplethysmograph (PPG) [2] and NIRS [5] recordings where a swinging hand or moving head can result in a change in the blood volume, or in electrocardiography (ECG) where the change in body position can alter the tissue conductivity conditions such that alterations in the potential distribution are affected [13].

The subject motion can also have an effect on the coupling of the electrode or optode. For systems such as ECG or EEG, a change in this interface changes the nature of the electrical coupling between them [15] and thus there is an additional component introduced to the potentials recorded at the bio-potential amplifier. For PPG and NIRS systems, a change in the position of the optodes causes a change in the optical path length of the transmitted or reflected light [7], thus causing a change in the intensity values detected. This inaccurate intensity level will, in turn, give inaccurate values for the oxygenated and de-oxygenated hemoglobin in the blood.

The different forms of movement generate different categories of artifact. It is therefore important to be able to distinguish between the different movements of the subject so as to be able to determine the type of artifact embedded on the underlying desired signal.

\section{ARTIFACT DETECTION}

As stated in Section II, motion artifact can originate from either the movement of the subject (inducing a physiological artifact) or from the movement of the sensor with respect to the body (inducing an instrumentation artifact). Therefore, if these individual movements can be distinguished and recorded then the areas of the data where the artifacts are likely to arise can be identified.

Accelerometers, due to their ease of use, have often been employed to detect motion for artifact detection. Some authors have used this accelerometer data to apply a quality of signal metric to the recorded data [13][6], thus showing where artifacts are likely to have occurred. Other authors have used the data as a reference for removal of the artifact using adaptive filters from ECG [9], PPG [2] [10] and NIRS [5].

In this paper we propose to use multiple accelerometers to detect the different forms of motion artifact. A single accelerometer is used to detect the movement of the subject in relation to the position that the physiological signal is being recorded. A second accelerometer is then used to measure the movement of the recording electrode or optode. With two separate sources of movement measures, it now becomes possible to detect the movement of the recording device with respect to the underlying tissue.

This new measurement makes it possible to not only detect when motion artifact is likely to have occurred, but what type of artifact it is likely to develop when it does occur.

\section{ARTIFACT CLASSIFICATION}

The proposed system allows us to detect and classify differential movement of electrodes or optodes in relation to the underlying tissue, as well as large body movement. This classification could then be used as both a signal quality metric, showing where the desired signal is embedded with noise, and as a reference to the areas of the signal that artifact removal should be employed. This reduces the amount of data that a technician would have to review and thus improves the efficiency of artifact detection post testing. Even if the artifacts in the signal have been reduced, using some artifact removal techniques, the information on where the signal has been modified can aid a technician in deciding to keep that section of data for post processing. Doing so reduces the risk of inaccurate data being used for postprocessing and thus reduces the influence that motion artifact currently possesses.

\section{CASE STUDY}

To emphasize the value of the classification algorithm described above, a number of tests were undertaken recording both NIRS and EEG from the dorsolateral prefrontal cortex. Both NIRS and EEG were recorded to show how artifacts are embedded differently on different physiological signals, and to show the advantage of dual modality recordings. The position of the NIRS optodes, EEG electrodes and accelerometers are shown in Fig. 1.

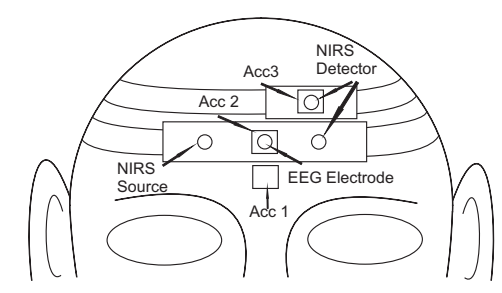

Fig. 1. Position of NIRS optodes, EEG electrodes and accelerometers on subject. 
In order to demonstrate the different forms of artifact, three separate experiments were performed. Each experiment consisted of a 10 second period of rest followed by 3 epochs of 20 seconds of activity followed by a final 20 seconds of rest.

Firstly, the subject was asked to move their head up and down continuously at a given rate which was kept constant over the duration of each activity period. The speed of the movement was increased, to a rate of approx 3, 6 and 8 times in 20 seconds, from one epoch to the following. During this test, the recording leads were not secured correctly so as to allow for an intermittent pulling on the leads when head movement occurred. This would cause some movement of the electrodes or optodes in relation to the underlying tissue. The subject was asked to complete two tests.

The second experiment was similar to the first but had the leads securely connected to the rear of the head so that very little tension was felt by the recording electrode and optodes. Again two tests were conducted.

Finally during the third experiment, the subject was asked to remain still for the duration. The headpiece holding the recording electrode and optodes were disturbed intermittently, for the duration of the experiment, so as to cause a differential movement between them and the scalp. Two tests were completed.
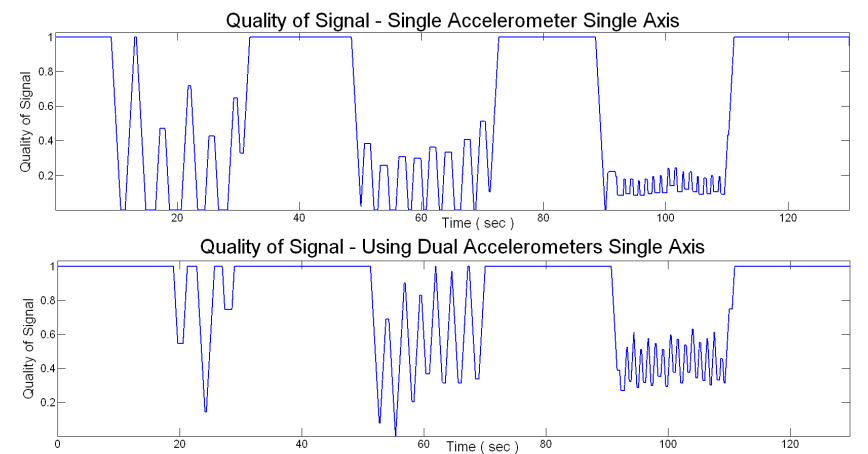

Fig. 2. Quality of Signal (QOS) metric determined using both single and dual accelerometers. A value of 1.0 demonstrates no motion detection, a value of 0 displays motion detected over all data samples in a one second epoch centered around the current sample.

Post testing, the accelerometer data is analyzed automatically using a motion detection algorithm. This algorithm detects movement which exceeds a threshold level, determined empirically, for both body and electrode/optode movement [13]. This algorithm is then used to create a quality of signal (QOS) metric for both movement types. An example of QOS acquired from a test from the first experiment is shown in Fig. 2. Finally these QOS data sets are used to create tagging data to classify the recorded signal as can be seen in Fig. 3.

As stated previously, NIRS is highly susceptible to noise due to movements of the head and movements of the optode in relation to the underlying tissue, whereas EEG is less susceptible to interference caused by head motion. Therefore when head movement is detected using the single accelerometer, this embeds an artifact on the NIR but the EEG remains
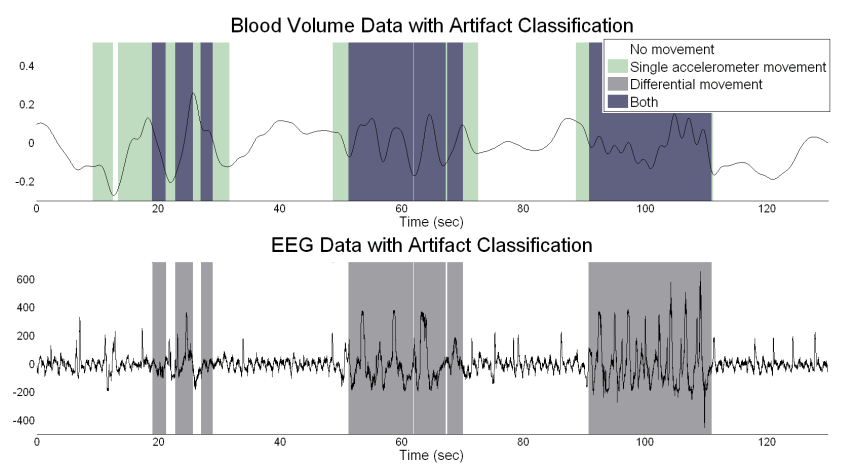

Fig. 3. Classification of artifacts embedded on physiological signals.

clear of contamination (Fig. 3). When differential movement is detected, the classifier shows the possibility of artifact on both signals . With both signals fully classified, it is possible to visually observe that there are indeed artifacts embedded on both signals where the classifier has shown.

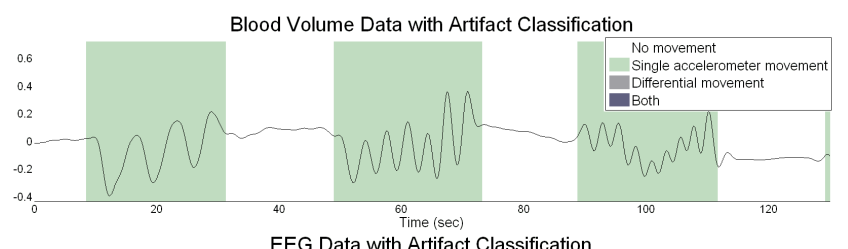

EEG Data with Artifact Classification

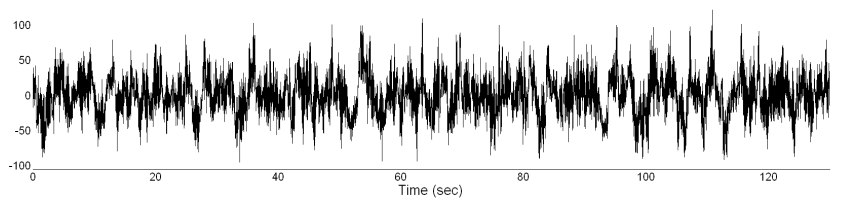

Fig. 4. Classification of data with blood volume artifact

Fig. 4 and 5 show the classification outputs from tests conducted during experiments 2 and 3 respectively. During experiment 2 , the authors aimed to produce purely head movement artifact to show firstly the classifier operating correctly and secondly to enforce the desire for dual modality measurement systems. It can be seen from Fig. 4 that although the blood volume data recorded by the NIRS system contains motion artefact, the corresponding EEG data does not, allowing accurate data to be recorded. The EEG signal observed is embedded with artifacts due to muscle and eye movements which must also be removed during post-processing. In experiment 3 (Fig. 5), the overall head movement was kept to a minimum and differential movement was created by pulling on the recording leads. This caused an artifact to be seen on both the NIRS and EEG data. As expected, the movement affects each signal in a different manner.

As further illustration of the workings of the classification system an adaptive FIR filter was implemented in MATLAB $\cap$. Fig. 6 shows the output of the adaptive filter along with the inputted blood volume recorded by the NIRS system with the accelerometer data used as a reference. It can be observed visually that the effect of the artifact embedded 


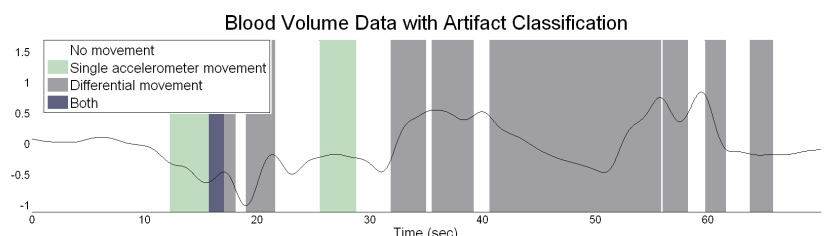

EEG Data with Artifact Classification

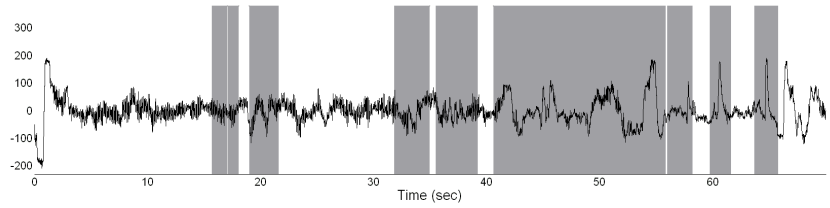

Fig. 5. Classification of data with artifact due to electrode/optode movement

on the blood volume data has been reduced significantly. This is to be expected as, from Fig. 4, it is known that the artifact is embedded due to the movement of the head recorded by the accelerometer. This output is similar to the result using adaptive filtering observed in [5].

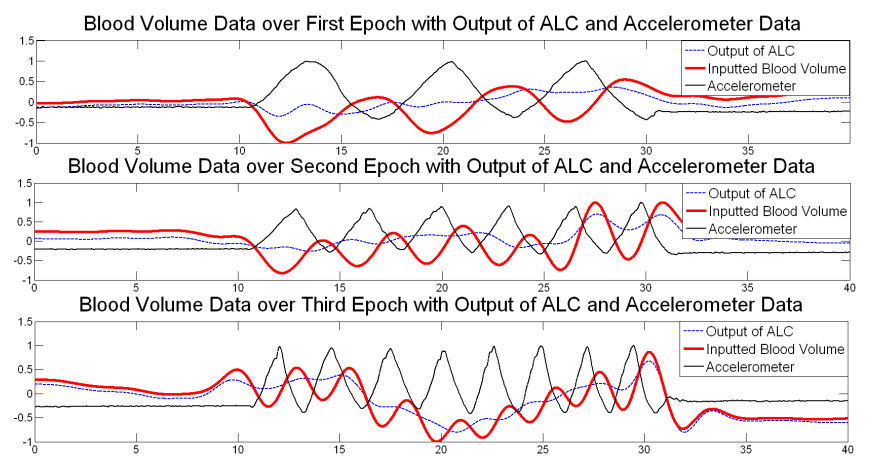

Fig. 6. Output of Adaptive Filter with Blood Pooling Artifact

However, as seen in Fig. 7, when the artifact is due to the movement of the optode, the adaptive filter is unable to remove the embedded artifact. As stated in [4] this artifact should be removed from the light intensity measurements recorded by the NIRS system prior to calculating the blood volume levels using the Modified Beer-Lambert Law (MBLL). Through the use of the information available from the classification algorithm above, the correct artifact removal techniques can be chosen with more certainty.

\section{CONCLUSION}

In this paper we present an approach for artifact detection, classification and tagging for ambulatory physiological signal monitoring using multiple accelerometers. Unlike previous classification algorithms, which are used to detect a single form of artifact, the ideas presented here allow for a tagging system which distinguishes between the two major forms of motion artifact: namely subject movement and sensor displacement relative to the underlying tissue. This classification data may be streamed with the recorded signals allowing better interpretation and post-processing of data.

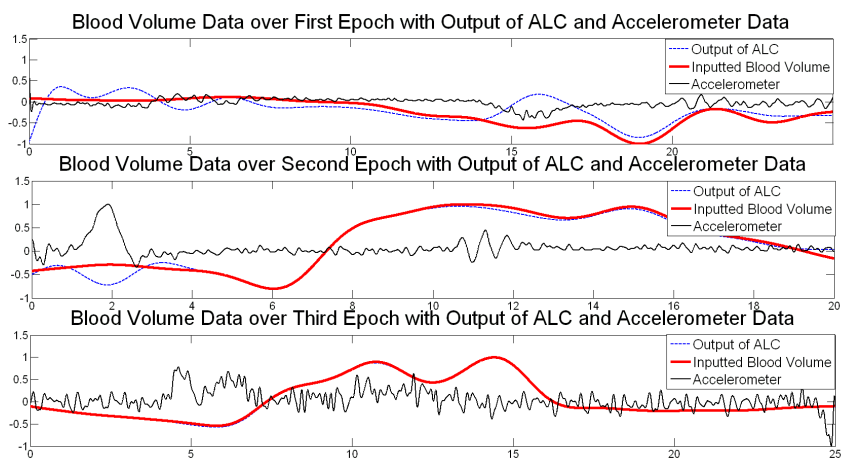

Fig. 7. Output of Adaptive Filter with Electrode/Optode movement Artifact

\section{REFERENCES}

[1] Joseph D. Bronzino. The Biomedical Engineering Handbook. CRC Press, 2000.

[2] G. Comtois and Y. Mendelson. A noise reference input to an adaptive filter algorithm for signal processing in a wearable pulse oximeter. In Bioengineering Conference, 2007. NEBC '07. IEEE 33rd Annual Northeast, pages 106-107, March 2007.

[3] A. Gautam, Young-Dong Lee, and Wan-Young Chung. Noise cancellation of electrocardiogram signal measured by wearable USN node. In Multisensor Fusion and Integration for Intelligent Systems, 2008. IEEE International Conference on, pages 154-157, Aug. 2008.

[4] Theodore J. Huppert, Solomon G. Diamond, Maria A. Franceschini, and David A. Boas. HomER: a review of time-series analysis methods for near-infrared spectroscopy of the brain. Appl. Opt., 48(10):D280D298, 2009.

[5] M. Izzetoglu, A. Devaraj, S. Bunce, and B. Onaral. Motion artifact cancellation in NIR spectroscopy using Wiener filtering. Biomedical Engineering, IEEE Transactions on, 52(5):934-938, May 2005.

[6] Y. Kishimoto, Y. Kutsuna, and K. Oguri. Detecting Motion Artifact ECG Noise During Sleeping by Means of a Tri-axis Accelerometer. In EMBS 2007. 29th Annual International Conference of the IEEE, pages 2669-2672, Aug. 2007.

[7] F. Matthews, B.A. Pearlmutter, T.E. Ward, C. Soraghan, and C. Markham. Hemodynamics for brain-computer interfaces. Signal Processing Magazine, IEEE, 25(1):87 -94, 2008.

[8] C. Papadelis, C. Kourtidou-Papadeli, P.D. Bamidis, I. Chouvarda, D. Koufogiannis, E. Bekiaris, and N. Maglaveras. Indicators of Sleepiness in an ambulatory EEG study of night driving. EMBS '06. 28th Annual International Conference of the IEEE, pages 6201-6204, 30 2006-Sept. 32006.

[9] M.A.D. Raya and L.G. Sison. Adaptive noise cancelling of motion artifact in stress ECG signals using accelerometer. In 24th Annual EMBS/BMES Conference, 2002. Proceedings of the Second Joint, volume 2, pages 1756-1757 Volume.2, 2002.

[10] A.R. Relente and L.G. Sison. Characterization and adaptive filtering of motion artifacts in pulse oximetry using accelerometers. In EMBS/BMES Conference, 2002. 24th Annual International Conference of the IEEE, volume 2, pages 1769-1770 vol.2, 2002.

[11] A. Shoeb, S. Schachter, D. Schomer, B. Bourgeois, S.T. Treves, and J. Guttag. Detecting seizure onset in the ambulatory setting: Demonstrating feasibility. EMBS 2005. 27th Annual International Conference, pages 3546-3550, Jan. 2005.

[12] Paul A. Sobotka, Joseph H. Mayer, Robert A. Bauernfeind, Charles Kanakis, and Kenneth M. Rosen. Arrhythmias documented by 24hour continuous ambulatory electrocardiographic monitoring in young women without apparent heart disease. American Heart Journal, 101(6):753 - 759, 1981.

[13] K. Sweeney, T. Ward, and S. McLoone. A simple bio-signals quality measure for in-home monitoring. In Proceedings of the 7th IASTED International Conference. IASTED, February 2010.

[14] E. Waterhouse. New horizons in ambulatory electroencephalography. Engineering in Medicine and Biology Magazine, IEEE, 22(3):74-80, May-June 2003.

[15] J. G. Webster. Medical Instrumentation, Application and Design. Wiley, 1998. 